\title{
Computer experiments on the onset of turbulence
}

T. H. Weisgraber, B. J. Alder

August 21, 2012

28th International Symposium on Rarefied Gas Dynamics Zaragoza, Spain July 9, 2012 through July 13, 2012 
This document was prepared as an account of work sponsored by an agency of the United States government. Neither the United States government nor Lawrence Livermore National Security, LLC, nor any of their employees makes any warranty, expressed or implied, or assumes any legal liability or responsibility for the accuracy, completeness, or usefulness of any information, apparatus, product, or process disclosed, or represents that its use would not infringe privately owned rights. Reference herein to any specific commercial product, process, or service by trade name, trademark, manufacturer, or otherwise does not necessarily constitute or imply its endorsement, recommendation, or favoring by the United States government or Lawrence Livermore National Security, LLC. The views and opinions of authors expressed herein do not necessarily state or reflect those of the United States government or Lawrence Livermore National Security, LLC, and shall not be used for advertising or product endorsement purposes. 


\title{
Computer Experiments on the Onset of Turbulence
}

\author{
Todd H. Weisgraber and Berni J. Alder \\ Lawrence Livermore National Laboratory, Livermore, CA 94550
}

\begin{abstract}
We are investigating if small amplitude distributed wall roughness, combined with fluctuations, could nucleate the onset of turbulence in bounded flows. Our direct numerical simulations of turbulent transition isolate the effects of the roughness since the only direct flow perturbations we consider are those due to natural hydrodynamic fluctuations. To properly resolve the range of length scales, we developed a conservative mesh refinement approach for the lattice-Boltzmann method.
\end{abstract}

Keywords: channel flow, turbulence, flow stability

PACS: $47.15 . \mathrm{Fe}$, 47.27.Cn

\section{BACKGROUND}

There is considerable experimental evidence that wall roughness is an important factor in the onset of turbulence. For one, turbulence in a pipe or between parallel plates is always observed to start near the wall. Furthermore, by carefully controlling inlet conditions and optically polishing the walls of a pipe, experimentalists have found that the onset of turbulence shifted from a Reynolds number of about $10^{3}$ to $10^{5}$ [1]. Finally, in the so called drag reduction problem, a small amount of polymer of the typical maximum size of the wall roughness of about a micron has been shown to delay the onset of turbulence $[2,3]$. Yet, theoretically or computationally, there have been few investigations of the effect of boundary conditions on flow stability.

Part of the reason for the lack of such studies, is a fundamental belief among fluid dynamicists that such small protrusions on the wall could not possibly matter. That is particularly true of the Russian school of applied mathematicians, led by Kolmogorov, who never questioned the nature of the boundary condition, namely the no-slip condition for a smooth wall. It is, of course, difficult experimentally, computationally or theoretically to deal with such a large range of length scales, from the nearly microscopic to the macroscopic and it is therefore convenient to ignore the very small scale. There exist well known dimensional arguments based on the viscous length scale, $\delta_{v}$, that rationalize that below a certain amplitude, $k$, the wall roughness can be neglected. This region, where $k^{+}=k / \delta_{v}<5$, is known as the viscous sub-layer where the viscous stress dominates over the turbulent stress. Experiments have shown that for amplitudes below the sub-layer, the fully developed turbulent friction is independent of the roughness [4], however the effect of these small amplitudes during transition are still unknown.

The theory of turbulence is considered to remain the biggest unsolved problem in classical physics and the culprit pertaining to the initial instability and the onset of transition may well be the use of idealized (smooth-wall) boundary conditions. The other possibility is that fluctuations are ignored in the mean field Navier-Stokes equations. Fluctuations also introduce, primarily, an even smaller length scale in the fluid flow equations than the rough boundaries and are therefore unlikely to initiate turbulence by themselves. The chances are good that the combination of fluctuation waves interacting with wall protrusions could resolve the turbulence initiation. In any case, we feel that this possibility should be computationally explored.

\section{RESULTS AND DISCUSSION}

For that purpose we have assembled in Fig. 1 linear stability studies of wall perturbations in pipe and channel flows. The figure shows the critical Reynolds number at which turbulence occurs for a given amplitude of the wall roughness. We first got into this field by studying the onset of turbulence in a corrugated pipe with no-slip boundary conditions. Heisenberg, in his doctoral thesis, showed that in a pipe with no-slip boundary conditions, the flow was stable at all Reynolds numbers for linear perturbations [8]. By introducing corrugations, a very unrealistic roughness, we showed through linear stability analysis that for a given Reynolds number, turbulence does occur at rather large amplitudes relative to the viscous sub-layer and the instability completely disappears at also rather large $(0.3 \%$ of 


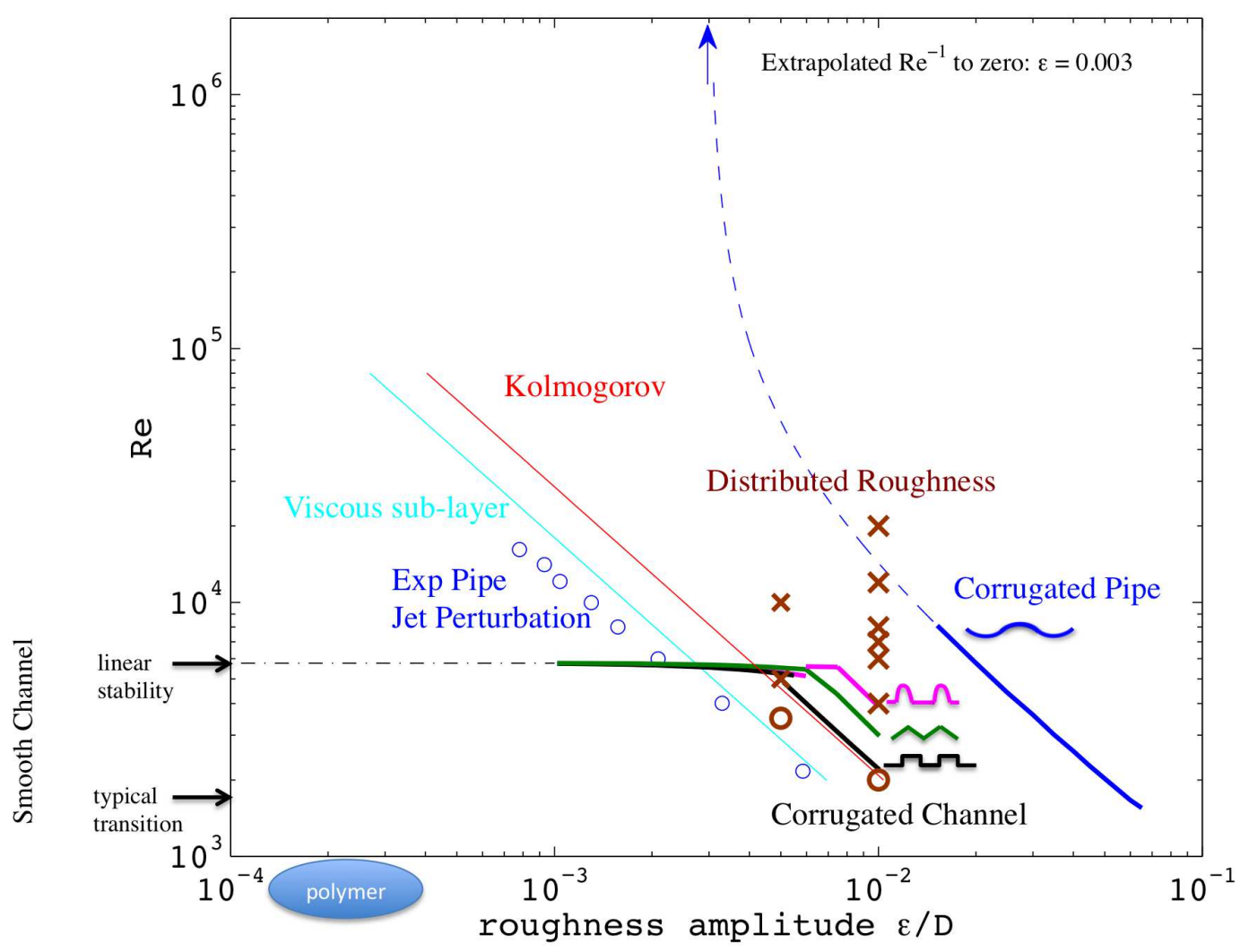

FIGURE 1. Critical transition Reynolds number as obtained from linear stability analysis of a corrugated pipe [5] and corrugated channels [6], with indicated roughness shapes. Open blue circles represent direct simulations of transition in a pipe initiated by injecting fluid at the wall [7]. In this case, $\varepsilon=\frac{\dot{m}_{\text {injection }}}{\dot{m}_{\text {pipe }}}$, where $\dot{m}$ is the flow rate. Brown points denote our direct simulations of rectangular distributed roughness in a channel $(x=$ turbulent transition, $\bigcirc=$ stable laminar flow). Also shown are the Kolmogorov scale and the thickness of the viscous sub-layer.

the pipe diameter) values [5]. We could determine this disappearance at infinite Reynolds number by extrapolating the amplitude linearly with one over the Reynolds number at large Reynolds numbers. We believe that such an extrapolation to infinite Reynolds number can be theoretically justified and leads to a minimum amplitude below which the flow is stable for any roughness, however, the amplitude value would be much lower for more realistic surfaces.

Other linear stability analysis have been carried out for parallel plates with periodic geometries of roughness [6,9], as indicated by the solid black, green, and lavender lines above the corrugated channel label in the figure. Rather insensitive to the shape of the roughness at also rather large amplitudes, the flow becomes linearly unstable at Reynolds number of about 6,000. For smooth parallel plates with no-slip boundary conditions the flow becomes unstable at that Reynolds number by linear stability analysis, as opposed to the pipe case, where it never becomes unstable. Experimentally, the flow becomes unstable both for typically commercially manufactured pipes or parallel plates at a Reynolds number of about 2,000 and there is the dilemma.

One could, of course, argue that the non-linearity is the problem, that is the linearization of Galilean invariance term in the Navier-Stokes equations in the stability analysis, but as we will show in our computer experiments, that is not the case. What is clear is that the viscous sub-layer scaling, which is nearly identical to the Kolmogorov scaling, corresponds to much larger Reynolds numbers than the linear stability curves at small amplitudes. The experiment which nearly agrees with these predictions (open blue circles) is one in which small holes are drilled into a pipe and 
fluid jets are injected into the holes to simulate roughness [7]. In this case, the analogy to the roughness amplitude is the ratio of the mass flow of the small jets to that of the mean flow.

Our preliminary computer experiments are direct simulations of instability solely due to perturbations initiated by three-dimensional distribution roughness elements. Crosses represent successful transitions, and open circles signify persistent laminar flow at rather large amplitudes. Fluctuations are not yet included. We appear to agree with linear stability analysis, but that is deceptive since we include non-linearities and expect to reach the experimental values of around a Reynolds number of 2,000 once fluctuations are included. The small amplitudes that are relevant experimentally and also for the drag reduction problem are indicated by the word polymer in the figure. The critical computer experiments need to be performed at that amplitude.

\section{The Lattice-Boltzmann Method}

Our computer experiments use the lattice-Boltzmann method (LBM), which we showed some time ago to exactly reproduce the Navier-Stokes momentum conservation equations when 19 different velocities are used. We believe the energy conservation equation is not necessary to invoke since temperature changes are small in turbulence onset and would require 64 velocities to satisfy the full Navier-Stokes equations [10]. The LBM is preferable over traditional Navier-Stokes solvers for several reasons. Both have the essential requirement that mesh refinement (MR) can be applied to span the length scales that have to be covered. A continuous space, generally used in particle dynamic schemes, like DSMC, would require an impossibly large number of particles, which even the largest imaginable computer could not handle. So far we have spanned a factor of $8=2^{3}$ in spatial resolution. This is accomplished by three stages of grid refinement, each by a factor of 2 . To get into the relevant regime $\left(\varepsilon / D=2 \times 10^{-4}\right)$, we need a further reduction by a factor of 32, easily achieved by using a factor of 4 in each stage of five refined meshes.

The advantage of using LBM over other Navier-Stokes solvers has to do with the ease of introducing boundary conditions. Since lattice-Boltzmann is basically a pseudo-particle method that evolves distribution functions for discrete velocities, the no-slip condition is enforced by simply reversing the outgoing distributions at the boundary. Another advantage of LBM is the ease with which fluctuations can be handled. When we tried to introduce fluctuations by the method suggested by Landau and Lifshitz into the Navier-Stokes equations, we found all existing Navier-Stokes solvers unstable. These solvers had been tested for stability at high wave number phenomena (shocks) and also for general low wave number situations. However, at intermediate wave numbers, as needed for fluctuations which contain all wave numbers, they were found to be unstable unless one went to higher order schemes (third order Runge-Kutta) and even then they were still not completely stable [11]. Lattice-Boltzmann experiences no such difficulties, one merely adds to the 19 distributions a fluctuating component.

However, even the Boltzmann equation is unstable when it is discretized. We found it even more unstable then the corresponding Navier-Stokes equation. When discretizing Boltzmann into lattice-Boltzmann one loses the H-theorem which Boltzmann proved so beautifully to be satisfied, leading to unconditional numerical stability. One can, however, reintroduce an $\mathrm{H}$-theorem in the LBM as a condition on each collision that guarantees numerical stability [12]. We have shown that this works in the Taylor-Green vortex problem, where without the imposition of an H-theorem other numerical schemes are unstable. This is an enormous advantage when studying instabilities. With other Navier-Stokes solvers one is never sure whether a numerical instability occurs rather than a physical one.

\section{CONCLUSION}

All the above assumes that the fluid dynamics equations are valid at the microscopic level, which we demonstrated some time ago, when we showed that Stokes law is valid at even shorter scales than a micron and for very short times $\left(10^{-12}\right.$ seconds) [13]. Thus flow past a sphere leads to vortex shedding even if the sphere is only a micron in diameter at a Reynolds number of between 20 and 40, independent of the size of the sphere till it gets to atomic dimensions. Thus, it is not hard to imagine that a protrusion of micron size can do the same, although, of course, the wall situation is not as favorable to vortex generation and, hence, a higher velocity or Reynolds number would be required.

This is an early progress report on the attempt to find the mechanism for the onset of turbulence. We completed the algorithm development to get mesh refinement conservative and valid to second order accuracy for the latticeBoltzmann method and are beginning runs to verify the physical hypothesis. We dedicate this paper to Graeme Bird. It was presented as the first lecture named in his honor. He pioneered in DSMC the particle solution of the Boltzmann 
equation, which makes its numerical solution immensely easier. Lattice-Boltzmann is a further development along the same line, namely a more coarse-grained discretization of the Boltzmann equation which is easy to implement and even faster. It is greatly satisfying that we find the approach that Bird initiated so extremely useful in tackling this fundamental problem.

\section{ACKNOWLEDGMENTS}

This work performed under the auspices of the U.S. Department of Energy by Lawrence Livermore National Laboratory under Contract DE-AC52-07NA27344.

\section{REFERENCES}

1. W. Pfenninger, "Transition in the inlet length of tubes at high Reynolds numbers," in Boundary layer and flow control, edited by G. Lachman, Pergamon, 1961, pp. 970-980.

2. W. White, and D. McEligot, J. Basic Eng. 92, 411-418 (1970).

3. W. Giles, and W. Pettit, Nature 216, 470-472 (1967).

4. H. Schlichting, and K. Gersten, Boundary-Layer Theory, Springer-Verlag, 2000, 8th edn.

5. D. L. Cotrell, G. B. McFadden, and B. J. Alder, Proc. Nat. Acad. Sci. USA 105, 428-430 (2008).

6. J. M. Floryan, Eur. J. Mech. B-Fluid 26, 305-329 (2007).

7. F. Mellibovsky, and A. Meseguer, Phys. Fluids 19, 044102 (2007).

8. W. Heisenberg, Annalen Der Physik 15, 577-627 (1924).

9. J. M. Floryan, Phys. Fluids 17, 044101 (2005).

10. G. R. McNamara, A. L. Garcia, and B. J. Alder, J. Stat. Phys. 81, 395-408 (1995).

11. A. Donev, E. Vanden-Eijnden, A. Garcia, and J. Bell, Comm. App. Math Comp. Sci. 5, 149-197 (2010).

12. S. Ansumali, I. V. Karlin, and H. C. Ottinger, Europhys. Lett. 63, 798-804 (2003).

13. B. J. Alder, and T. E. Wainwright, Phys. Rev. A 1, 18-21 (1970). 